\title{
Sources, mechanisms, and fate of steroid estrogens in wastewater treatment plants: a mini review
}

\begin{abstract}
Steroid estrogens, such as estrone (E1), 17 $\beta$-estradiol (E2), estriol (E3), and 17 $\alpha$ ethinylestradiol (EE2), are natural and synthetic hormones released into the environment through incomplete sewage discharge. This review focuses on the sources of steroid estrogens in wastewater treatment plants (WWTPs). The mechanisms and fate of steroid estrogens throughout the entire wastewater treatment system are also discussed, and relevant information on regulatory aspects is given. Municipal, pharmaceutical industry, and hospitals are the main sources of steroid estrogens that enter WWTPs. A typical WWTP comprises primary, secondary, and tertiary treatment units. Sorption and biodegradation are the main mechanisms for removal of steroid estrogens from WWTPs. The fate of steroid estrogens in WWTPs depends on the types of wastewater treatment systems. Steroid estrogens in the primary treatment unit are removed by sorption onto primary sludge, followed by sorption onto micro-flocs and biodegradation by microbes in the secondary treatment unit. Tertiary treatment employs nitrification, chlorination, or UV disinfection to improve the quality of the secondary effluent. Activated sludge treatment systems for steroid estrogens exhibit a removal efficiency of up to $100 \%$, which is higher than that of the trickling filter treatment system (up to 75\%). Moreover, the removal efficiency of advance treatment systems exceeds 90\%. Regulatory aspects related to steroid estrogens are established, especially in the European Union. Japan is the only Asian country that implements a screening program and is actively involved in endocrine disruptor testing and assessment. This review improves our understanding of steroid estrogens in WWTPs, proposes main areas to be improved, and provides current knowledge on steroid estrogens in WWTPs for sustainable development.
\end{abstract}

Keyword: Fate; Mechanisms; Regulatory; Sources; Steroid estrogens; Wastewater treatment system 
